\title{
Conclusiones del estudio exploratorio en torno a los alcances culturales derivados de la introducción de tecnologías de información en escuelas rurales.
}

\author{
Miguel Angel Arredondo Jeldes \\ Programa Interdisciplinario de Investigación \\ en Educación (PIIE), Chile.
}

\section{Introducción}

Entre las facetas distintas del fenómeno estudiado podemos mencionar las transformaciones de lo local ante la irrupción de lo global, las implicancias de potenciales cambios que se pueden producir al interior de la escuela tradicional, los repercusiones para los distintos actores (alumnos, profesores, directivos) ante esta nueva realidad.

Sobre lo anterior nos parece conveniente plantear que en el marco de la sociedad contemporánea, globalizada e intercomunicada se han naturalizado socialmente ciertas concepciones sobre el desarrollo que parecen estar en un nivel más alto que el de simples supuestos a ser debatidos. En países como los nuestros, donde cotidianamente se tematizan como problemas la pobreza y la falta de oportunidades para mejorar la calidad de vida de amplios sectores de la población se vislumbran ciertas fórmulas que se han consolidado en el imaginario social de nuestra región como vías de solución para estos problemas. Una de estas vías de solución es entender a la educación como un motor que posibilita la movilidad social, única posibilidad de que el grueso de la población acceda a mejores estándares de vida. "La educación en la llamada sociedad postindustrial -compleja y ambivalente- aparece como el medio predilecto para asegurar mañana lo que hoy no se ha logrado: un dinamismo productivo con equidad social y una democracia basada en una ciudadanía sin exclusiones. Esta expectativa encierra el peligro de una futura decepción, pues tales objetivos sólo pueden ser logrados a través de un vasto esfuerzo sistémico, del cual el sistema educativo puede constituir una parte importante, pero en ningún caso puede ofrecer las 'llaves del reino'." (Hopenhayn y Ottone. Pág: 34)

A esto se suma el convencimiento de que uno de los condicionantes históricos y estructurales que han perpetuado el retraso de la región es el bajo desarrollo tecnológico de sus aparatos productivos, que en vez de posibilitar la innovación y la creación de valor agregado a sus productos, no son más que meros receptores de segundo orden, que en buenas cuentas no hace más que mantener este estatus económico desmejorado.

Así nos encontramos frente a dos utopías que nos hablan de la superación de la pobreza: la pedagógica y la tecnológica. La primera postula que con una mayor educación las personas de estratos socioeconómico bajo tendrán acceso a una mejor movilidad social y con más herramientas para convivir dentro de una sociedad en constante cambio. Por su parte, la utopía tecnológica se refiere a que el subdesarrollo puede ser superado en la medida que se intensifica el uso de tecnologías en el campo productivo como de la información.

El problema con el que nos enfrentamos es cuando estas "apuestas" o decisiones, que tienen un carácter social e histórico (y que se traducen en políticas públicas como la evidenciada por el proyecto "Red Enlaces"), toman un sello de verdades absolutas e incuestionables. A eso hay que oponer al 
menos una postura más crítica y que revele algunas de las contradicciones y supuestos que se manejan en la introducción de las Tecnologías de información y comunicación (TIC) en las escuelas, y que son foco de nuestra investigación.

\section{El proyecto "Red Enlaces". Objetivos y supuestos de base.}

Un primer paso para poder aclarar en qué consisten los conceptos que sustentan a la "Red Enlaces" requiere hacer una breve relación de hechos que ordene cronológicamente cual ha sido la progresión que ha manifestado este proceso en particular. Los comienzos de esta iniciativa pueden ser rastreados hasta el año 1992 cuando el ministerio de educación chileno hace explícita la necesidad de construir "una Red Educacional Nacional entre todas las escuelas y liceos subvencionados del país". Esta incipiente primera etapa tenía contemplado un plan piloto concentrado en una docena de escuelas de la capital Santiago, para luego extenderse a la novena región (división político-administrativa con el mayor índice de población indígena) donde se incluye un total de 100 escuelas a esta etapa de incipiente desarrollo. Este período de prueba se extiende hasta 1995 donde comienza a tomar forma un verdadero plan de crecimiento a nivel nacional. Desde este año en adelante ya se plantea la factibilidad de que los recursos invertidos en las TIC's (equipos, software y conexión a Internet) deberían estar disponibles en todas las escuelas de Chile dentro de un plazo no superior a los cinco años. Conforme a este plan, desde el año 1996 puede anotarse un punto de inflexión en términos de cobertura que, ahora en términos exponenciales, inicia definitivamente su expansión a nivel nacional. De forma tal que para el año 1998 la informática ya es considerada como parte integrante del currículum para la educación secundaria bajo el estatus de "objetivo transversal", es decir, se considera que el trabajo con las TIC's tiene ramificaciones hacia todos los aspectos de la formación recibida por los alumnos. Tal como queda registrado en este gráfico, la progresión que ha manifestado la "Red Enlaces" ha sido la siguiente:

\section{Establecimientos en Enlaces: Expansión}

6.000

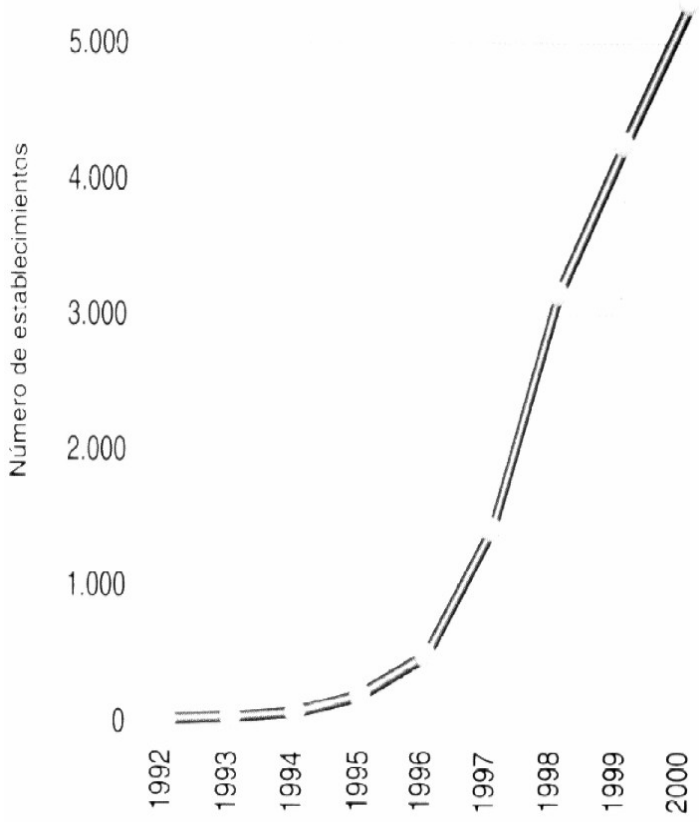

(Fuente: Ministerio de Educación)

Dados estos resultados puede sostenerse que en cifras gruesas la dotación de equipamiento informático (computadores, software, Internet y periféricos) en el sistema educacional chileno ha conseguido un amplio éxito. Aunque todavía es muy evidente la diferencia en la disponibilidad de estas nuevas tecnologías que hay entre las escuelas ubicadas en los grandes centros urbanos y las zonas rurales más apartadas, no es menos cierto que paulatinamente las escuelas rurales están siendo 
incorporadas a un plan de integración que en mediano plazo debería superar estas diferencias de equipamiento. Una mirada general nos indica que las TIC's de uno u otro modo se están haciendo parte habitual del paisaje escolar. Esto último se verifica en la notoriedad mediática que ha alcanzado todo lo que esté relacionado con la irrupción de las TIC en la sociedad y que puede verse en las expectativas que generan tanto en profesores como alumnos frente a esta nueva realidad.

Ciertamente esta modernización en la educación tiene un sustento conceptual que relaciona el trabajo de orden técnico; como es el establecimiento de una red informática, con los fines, necesidades y desafíos que enfrenta actualmente la educación. Evidentemente esta inversión en gran escala no podría operar sin contar con un ideario que defina con precisión cuáles son los parámetros básicos que guían todo el accionar de la "Red Enlaces" como programa gubernamental.

Estas directrices lo que intentan es normar algunas de las principales cuestiones que emergen en paralelo a la introducción de tecnología en educación, por ejemplo; al determinar cuál es la orientación pedagógica que se pretender dar a las TIC's una vez que estas ingresan a la sala de clases, qué tipo de nuevos conocimientos y habilidades pueden obtener los alumnos gracias a las TIC's, de qué forma la gestión de la escuela se verá beneficiada mediante el uso de la informática, la idea de que el docente puede apoyarse en los recursos disponibles en la red para complementar la realización de su clase, etc. Es más, uno de los soportes conceptuales del proyecto Enlaces "considera a las tecnologías de información y comunicaciones como una herramienta al servicio de las personas, de los protagonistas del proceso de enseñanza y aprendizaje: alumnos, profesores, directivos, sostenedores y apoderados de los establecimientos educacionales. (...) Tener como meta no sólo dotar de computadores a las escuelas y liceos, sino que integrarlos a una red educacional que les permitiera estar comunicados entre sí y con el mundo, e intercambiar ideas y experiencias, independientemente de la región o comuna donde se situaran. Esto apunta a cumplir con uno de los objetivos que persigue la reforma educacional: lograr una mayor equidad en las oportunidades de los niños y jóvenes de acceder a una educación de mejor calidad.”[1] De lo anteriormente señalado se desprende un supuesto que sin ser explicitado marca la dirección hacia donde se pretende llevar este proceso de cambio tecnológico, con esto nos referimos a la gran cuota de confianza existente en que las tecnologías de la información tienen un potencial transformador que opera por sí mismo.

Esta cualidad que tendrían las TIC's, traspasada al ámbito de la educación, permitiría aumentar la calidad de la educación, asegurar la equidad de ésta de momento que todos los alumnos comparten la misma "ventana de acceso" al mundo que es Internet, hacer de los alumnos "ciudadanos del mundo" con capacidad de comprender y ser partícipes del proceso de globalización. En la perspectiva del núcleo planificador del proyecto "Red Enlaces" las TIC's no sólo se constituyen en una herramienta con una tremenda gravitación en plano pedagógico sino que también están relacionadas con el tipo de vida que experimentarán los niños y niñas que hoy están recibiendo esta formación en informática. Esta "revolución educativa tendrá que ver con la educación permanente (life-long learning), la educación a distancia y la educación basada en las TIC's. Los países que no logren abordar este cambio verán ensanchar la brecha de conocimiento y de capacidades tecnológicas con las partes más dinámicas del mundo globalizados y no podrán formar parte de la economía basada en conocimientos y de la sociedad de la información."[2] Según estos argumentos, la introducción de tecnologías de la información en el sistema educacional viene a constituir la respuesta a la urgente necesidad de que la educación impartida en Chile logre adecuarse a los requerimientos que supone el proceso de globalización.

La investigación cuyas conclusiones presentamos se orientó por preguntas tales como: ¿Esta tecnología se incorpora realmente como una nueva herramienta pedagógica a la escuela?, ¿Qué efectos culturales se producen en el ámbito rural-local a partir de la introducción de esta nueva tecnología globalizadora?, ¿Qué sucede en la relación profesor-alumno, en la escuela rural cuando se introducen las tecnologías de la información y las innovaciones pedagógicas que estas conllevan?, ¿Cómo reacciona el alumno frente a este nuevo recurso educativo?, ¿Cuáles son las expresiones y sentidos que el alumno puede construir en su relación con la tecnología? 
Estas interrogantes plantean sin duda cuestionamientos teóricos y prácticos a los supuestos de Enlaces. Nos parece que es necesario profundizar lo que las cifras de cobertura y número de profesores capacitados en las TIC's conciben como un avance notable en el mejoramiento de la calidad educativa y en verdaderas herramientas que posibiliten la superación de la pobreza. Pues para que las TIC's tengan un desarrollo que propicie un verdadero impacto en la disminución de la brecha de la equidad es imprescindible agregar tanto una dimensión más cultural como también un conocimiento cabal sobre los verdaderos usos sociales y prácticas que se desarrolla en la escuela en relación a la incorporación de las TIC's.

Nuestra investigación se desarrolló en el contexto de localidades rurales en sectores de pobreza con el objeto de conocer las prácticas sociales que están ligadas al uso de las TIC en las escuelas rurales. La comuna de Maule[3] fue seleccionada en tanto cumplía con el requisito de tener escuelas básicas integradas al proyecto "Red Enlaces".

\section{Reflexiones y desafíos pendientes.}

En el transcurso de la presente investigación un primer hecho que se ha podido constatar, es la distancia entre una construcción de carácter utópico que rodea a la introducción de las TIC's y una realidad concreta en la cual éstas entran a interactuar con actores concretos que "reciben" estas innovaciones en su entorno cotidiano.

Esta utopía a la que hacemos mención, se refiere a la promesa que implica crear un nuevo tipo de sociedad sustentada en el desarrollo tecnológico (en especial de las comunicaciones) y sus aplicaciones a las dinámicas sociales. Las dos grandes promesas involucradas en esta utopía tienen que ver, en primer lugar, con la eliminación de la distancia tanto en sus aspectos espacio-temporales como en sus aristas de orden sociocultural. Es decir, que en la sociedad de la información la tecnología permite generar un flujo de comunicaciones tal que permitiría soslayar las fronteras naturales que dificultaban la relación entre las personas y las sociedades.

En segundo lugar, y relacionado con lo anterior, también se plantea que el conocimiento asumiría una mayor cercanía, dejando de ser un "monopolio" de unos pocos, al presentar, gracias a las TIC's, múltiples entradas posibles a éste. Esto debido esencialmente a que el acceso a las fuentes de este conocimiento se hace más expedito e instantáneo (un ejemplo al que se recurre constantemente se encuentra en los sitios Web que permiten observar las actividades de la NASA o ingresar a los principales museos del mundo). Todo lo cual sustentaría la construcción de una sociedad en donde el eje central es el "conocimiento", a diferencia de la sociedad industrial donde lo que primaba era la técnica. Esta característica sería el sustrato básico del carácter esencialmente democrático de la sociedad de la información, en la cual todos podrían participar del conocimiento.

Ahora bien, como toda utopía esta se ve cuestionada por la realidad, en el sentido de que muchas de las esperanzas que se centran en la TIC's se ven enfrentadas a las complejidades y contradicciones de la realidad social. Sin ir más lejos, el concepto de "brecha digital" es claramente insuficiente para dar cuenta de las dinámicas que emergen desde los intentos de extender las lógicas de la sociedad de la información a toda la sociedad en su conjunto. En este sentido la superación de la "brecha digital" siempre se ha entendido como la extensión masiva de la tecnología de información y comunicación, midiéndose en la cantidad de computadores per cápita, en el número de celulares, los metros de fibra óptica, etc. Desconoce que tras estos procesos se manifiestan falencias de orden cultural, a lo cual apunta el concepto de "brecha digital de segundo orden". Esta se refiere a las complejidades de orden social que dificultan que la tecnología asuma un real significado, tendiente a facilitar cambios sociales que de ellas se esperan. Y pudiendo incluso profundizar las distancias sociales que en teoría la tecnología debería superar.

Lo anterior apunta a que no basta con la simple presencia de infraestructura de TIC's para lograr que estas contradicciones expuestas se eliminen. Para que la "brecha digital de segundo orden" pueda ser eliminada se requiere de un proceso de "apropiación cultural" de las TIC's. Esto es que los distintos 
actores sociales puedan dimensionar a las TIC's como algo propio y útil para su mundo. Lo que quiere decir que no sólo hay que adaptarse a la tecnología sino que esta también debe tener la capacidad de adaptarse a los actores que la requieren.

Dentro de este proceso de "apropiación de las TIC's" la escuela, por su función social asume un rol central como agente privilegiado para liderar y llevar a cabo este proceso. Pero para que se logre esto, la escuela como institución debe desarrollar en su interior un proceso de apropiación, el cual hemos definido como "integración". La integración da cuenta de las diversas estrategias y condiciones que son necesarias por parte de la escuela para apropiarse ella y sus miembros de estas nuevas tecnologías. De ahí que la integración implica la generación de nuevas metodologías de trabajo y gestión, involucrando a profesores, directivos, alumnos y la comunidad.

Todo lo anterior da cuenta que para lograr el aspecto, a nuestro juicio, más central de la utopía de la sociedad de la información, que es sustentar una mayor democratización de la sociedad a través de expandir la posibilidades de comunicación y liberalización de la información, se requiere no sólo de su disponibilidad sino que, y sobre todo, de desarrollar dinámicas que potencien la apropiación cultural. Por lo cual el problema se juega, entre otros elementos, en la posibilidad de generar dinámicas de consumo cultural que respondan a la construcción de sentido necesaria para toda construcción democrática.

\section{Preguntas de discusión}

\section{1 ¿CÓMO LA INTRODUCCIÓN DE LAS TIC'S PUEDE AYUDAR A LOGRAR EL OBJETIVO DE TENER UNA MEJOR EDUCACIÓN?}

Plantear esta pregunta a estas alturas, justamente cuando un porcentaje importante de los establecimientos del sistema público de educación forma parte de este proceso, puede resultar una pregunta incómoda para planificadores, autoridades, proveedores, etc. El punto que interesa dejar bien establecido a través de esta pregunta es que si bien las TIC's suponen un nuevo escenario para la escuela, más aún para la escuela rural, ello no significa que gracias a su sola presencia comiencen a operar las transformaciones y se alcancen los beneficios que permanentemente se prometen a raíz del uso de las tecnología de la información en el ámbito de la educación.

Hay muchos factores que han sido abordados en este trabajo que limitan el impacto de las TIC's (entre otros las resistencias del profesorado, uso orientado hacia lo lúdico, sistema de premios y castigos) pero a nuestro juicio un elemento que es crucial se refiere a la incapacidad de advertir que las TIC's se constituyen en algo mucho más complejo que una simple técnica puesta a disposición de la escuela. Una técnica que al igual que la televisión y el video educativo sigue teniendo un carácter esencialmente unidireccional, en donde le profesor o el alumno tienen una muy limitada capacidad de intervención sobre estos medios. Por el contrario las TIC's si representan una tecnología que efectivamente permite que el usuario pueda "manipular" con mucha mayor libertad los contenidos y las aplicaciones que se encuentran disponibles tanto en Internet como en el software instalado. Al entender a las TIC's como una simple técnica recién instalada también se tiende a abordarlas como un bien material, el cual significa atender las necesidades inmediatas que esta bien requiere (básicamente que no se dañe) por sobre plantearse las potencialidades y objetivos que gracias a las TIC's pueden lograrse.

Esta última combinación de factores nos remite al punto crítico que hasta este momento no ha sido abordado en toda su amplitud y complejidad por las políticas públicas encargadas de la extensión de las TIC's en la educación. Y el cual ha sido uno de los conceptos claves tratados a lo largo de este trabajo; este se refiere a la apropiación de la tecnología. Entendida como la generación de sentido en torno a la utilización y aprendizaje de las TIC's apara estos nuevos usuarios, es decir, que estas nuevas tecnologías se transformen en una necesidad verdaderamente sentida, desde la cual su uso finalmente se hará más intenso. En otras palabras, desde la escuela rural aún no se dimensiona adecuadamente por qué razón se ha hecho esta inversión en equipamiento computacional ni tampoco está muy claro 
como podrían ayudar al trabajo escolar. Sin estas coordenadas básicas el impacto que las TIC's tienen sobre los procesos educativos sigue siendo muy cuestionable.

Sobre la base de lo anterior, sólo existiendo un alto grado de apropiación de las nuevas tecnologías es posible comenzar a plantear la forma en que las TIC's pueden hacer un aporte sustantivo al mejoramiento de la educación. Sólo bajo estas condiciones es posible plantear dentro de un terreno más seguro la cuestión sobre la "brecha digital" y las consecuencias que ella puede acarrear para los países (y, claro, su población) que se ubican dentro del lado “equivocado” de este fenómeno.

Otro punto que debiera cobrar más importancia para el debate tiene relación con los contenidos de carácter educacional para los cuales las TIC's son el medio más indicado para transmitirlos y otros que siguen siendo valiosos a pesar de su formato tradicional con el que siguen siendo presentados. Si bien las TIC's presentan formas novedosas de recibir y procesar nuevos conocimientos, por ejemplo, a través de aplicaciones muy poco utilizadas hasta ahora como los simuladores (de física, química, biología, etc) ellas no pueden reemplazar la adquisición de conocimientos que no forman parte de este nuevo acervo cultural ligado a la informática. Con esto nos referimos a aspectos centrales en la formación general de cualquier alumno que, junto con requerir un buen manejo de las TIC's para verse integrado a la llamada "sociedad de la información", debe tener un repertorio de conocimientos cuyo medio de transmisión no son las TIC's. Con esto nos referimos a conocimientos que siempre han sido considerados como valiosos y necesarios para la formación todo alumno o alumna, un ejemplo que no es menor se refiere a la lectura de libros clásicos de la literatura, los que para cualquier persona son un capital cultural que hasta ahora no puede ser entregado por otro medio que no sea el libro. Para este tipo de conocimientos tan valioso el formato electrónico sigue siendo un medio claramente insuficiente.

Esto tiene que ver con que el proceso de apropiación e introducción (una vez alcanzada esta condición) de las TIC's no caiga en el error de creer que todo conocimiento obligatoriamente debe ser traspasado al formato de las tecnologías de información y comunicación. Hay muchas formas de comunicación que no por ser "tradicionales" han perdido fuerza y eficacia como ocurre en especial con el libro.

En suma una vez superadas las barreras que implica la brecha digital de segundo orden y lograda la apropiación de la tecnología por parte de los actores educacionales, ello no significa que para hacer su aporte a una mejor educación las TIC's tengan que convertirse en la única forma de transmitir y adquirir conocimiento bajo la estructura escolar.

\section{2. ¿QUÉ HA SIGNIFICADO LA PRESENCIA DE LAS TIC'S PARA LA ESCUELA?}

Indudablemente, y aun cuando parezca obvio afirmarlo, las TIC's han constituido una novedad para la escuela. El primer paso para poder entender el proceso que se ha venido desenvolviendo en estas escuelas, es la constatación de que las TIC's son algo "nuevo", algo que la escuela, como institución, no había concebido y para lo cual no estaba diseñada. No contaba con antecedentes históricos de experiencias similares que le fueran útiles para poder establecer estrategias que le permitieran asumir a las TIC's (con todo lo que ellas conllevan) de una forma no disruptiva[4]. En este sentido lo "nuevo" tiene una íntima relación con lo distinto.

Ahora bien, la idea de lo "nuevo" no es unívoca, como concepto de valoración es ambiguo[5], ya que "nuevo" puede tener tanto un sentido de innovación, de actualidad, de naciente, todos con un carácter positivo, como a la vez también hacer referencia a lo desconocido, a lo extraño con un sentido quizás no necesariamente negativo, pero sí de incertidumbre. Es por lo mismo que sea entonces en esta dimensión de la novedad, del cómo esta va a ser asumida, en donde se juegue gran parte de la capacidad transformadora que puedan llegar a significar las TIC's en las escuelas.

Esta ambigüedad del concepto tiene mucho que ver con la visión del mundo que se tenga, es por ende un aspecto de una construcción ideológica. Así por ejemplo, dentro de los parámetros de la 
Modernidad la idea de lo "nuevo" se constituye en un sinónimo de positivo, si es "nuevo" es bueno, mejor que lo viejo. Esta concepción no es un mero capricho, si no que sustenta a uno de los pilares fundamentales del discurso de la modernidad, el cual es la idea de Progreso, permanente e ilimitado, en el cual el hombre siempre va a ir superándose, contraponiendo un promisorio futuro frente a un pasado oscuro.

Dentro de esta concepción teleológica de la vida, el discurso de la Modernidad se traduce al ámbito de la tecnología en un permanente anhelo por la innovación, por la generación de nuevas herramientas, de nuevos materiales, etc., donde el último invento siempre será concebido como el mejor[6].

Pero ciertamente, como todo el discurso de la Modernidad, esta mirada optimista tiene su contracara, lo nuevo, como se mencionó más arriba, también hace referencia a lo distinto, a lo extraño y por ende es fuente de recelo, la permanente innovación puede generar incertidumbre, y lo "nuevo" transformarse entonces en una amenaza para la estabilidad, así el optimismo del Progreso tiene su lado negativo en la incertidumbre respecto al futuro.

Así innovación e incertidumbre son dos dimensiones de un mismo proceso, y como las dos caras de una misma moneda son inseparables y, por lo tanto, no pueden ser ignoradas a la hora de pretender implementar transformaciones tan ambiciosas que busquen instaurar lo "nuevo" en una institución.

Lo importante de constatar este carácter de ambigüedad que se genera entorno a lo "nuevo", es que permite entender que ante la innovaciones existen distintas formas de reaccionar, lo "nuevo" es asumido de diversas maneras, dependiendo de las personas y las instituciones, las que pueden variar dependiendo de una multiplicidad de factores, por lo que cualquier innovación puede se asumida de las más diversas maneras. Así por ejemplo en el caso de las escuelas observadas, cada profesor tenía una lectura propia respecto al significado de la introducción de TIC's, y reaccionaba por ende de diversa manera[7]. En una misma escuela había quienes manifestaban una gran confianza y optimismo y otros que derechamente recelaban de esta tecnología. Lo mismo puede extrapolarse al nivel de las escuelas, entre las cuales también existen diferencias respecto a cómo afrontar la innovación TIC's. La instituciones al igual que las personas tiene diversas maneras de reaccionar ante cualquier innovación y de asumir lo "nuevo".

Así entonces la introducción de las TIC's en las escuelas observadas, ha desencadenado toda una dinámica de reacciones diversas ante la presencia de un elemento extraño para ese mundo delimitado que constituye la escuela en el entorno rural.

Las TIC's en este sentido han, en cierta medida, alterado algunas dinámicas tradicionales y generado otras nuevas, han introducido optimismo y desconfianza, han creado nuevas figuras a la vez que mantienen a otras intactas, en definitiva, y esto es importante recalcarlo, han hecho reaccionar a los distintos actores que constituyen la escuela, no los ha dejado indiferentes, los ha interpelado y obligado a establecer una posición respecto a las posibilidades de innovación dentro de la escuela.

Ahora bien lo anterior deja en claro que aún existe una gran falencia y por ende un desafío, en el proceso de introducción de las TIC's. Esta se relaciona con la necesidad superar la incertidumbre que genera la presencia de este elemento nuevo, lo cual pasa, necesariamente, por el hecho de dejar de ser un elemento extraño[8] para la escuela y sus miembros.

La pista para lograr ésto la dan los propios profesores. Lo "nuevo" sólo logrará constituirse en una innovación, o sea en asumir un carácter positivo, cuando logre generar un sentido para su uso, una razón de ser para ese lugar y momento preciso. Es a partir de ese punto que las dinámicas que se establecen entorno a las TIC's van a poder incluir a la institución completa y a todos sus miembros, superando el carácter parcial y delimitado que tienen hoy en día[9].

El desafío, en este sentido, consiste precisamente en desarrollar la innovación, esto quiere decir, establecer un sentido concreto, dentro de la escuela respecto a la potencialidad del trabajo con las 
TIC's. Esto por que ese sentimiento positivo respecto a lo "nuevo" no es automático ni evidente, y no basta con las alusiones pomposas a "sociedades informáticas" como para eliminar todo rasgo de incertidumbre y desconfianza que pueda generarse en los profesores, para eso es necesario se sientan participes de la innovación y no que esta los vaya a dejar de lado. Son ellos quienes precisamente tienen que ser los más convencidos en este proceso por lo cual no pueden tenerle desconfianza.

\section{3. ¿QUÉ HABILIDADES Y ACTITUDES REQUIERE LA PRESENCIA DE LAS TIC'S EN LA ESCUELA?}

La posibilidad de que las TIC's se transformen en herramientas para producir un cambio social y cultural en la educación chilena, como hemos visto, requiere de la motivación (relacionada con las expectativas) y la apropiación que los actores involucrados puedan llegar a desarrollar. Este proceso de resignificación constante en que sobre todo los docentes parecen estar más tensionados, establece un cuestionamiento abierto al tema de cuáles son las habilidades y actitudes que se requieren en este escenario cambiante para llevar a buen puerto la introducción de TIC's en las escuelas.

El hecho de que la escuela sea conceptualizada como una institución tradicional y que, sin duda, las TIC's sean visualizadas como lo innovador, gatilla conflictos en cuanto a la organización, la estructura, las relaciones y las valoraciones que se encuentran presentes en la comunidad educativa, pero por sobre todo, en la lógica docente.

Como vimos en capítulos precedentes, la posición del profesor frente a la tecnología y a sus alumnos, puede verse reflejado en tres tipos; el facilitador, que además de dominar correctamente la tecnología, le entrega a sus alumnos las herramientas necesarias para manejar los computadores y lejos de controlarlos y restringirlos, los orienta y les concede un grado de libertad y experimentación. Otro tipo es el ausente, que ante su falta de seguridad con la tecnología o su escasa motivación deja a los alumnos sin orientación en el trabajo con los computadores, o en el peor de los casos, prefiere no entrar a la sala de computación. El tercer tipo de actitud que puede asumir el docente es la de dirigista, aquel que al tener la percepción de que sus conocimientos son reducidos, manifiesta su inseguridad controlando en grado sumo los comportamientos y actitudes de los alumnos frente al computador.

Esta tipología sin duda refleja distintos grados de apropiación de la tecnología pero, también da cuenta, de distintos niveles de actitudes y conocimientos en los encargados de llevar a cabo la potenciación de lo pedagógico, mediado por lo tecnológico.

Niveles tan distintos de aproximación a la tecnología por parte de los docentes lleva a preguntarse, cuáles son las habilidades y las capacidades que requieren procesos como el llevado adelante por la "Red Enlaces" para una utilización cabal de las TIC's y para el desarrollo de un capital transformador en las comunidades donde se han asentado estos nuevos medios.

En este plano el tema de las concepciones tradicionales de lo pedagógico, al parecer se enfrenta a un punto de inflexión ante el cuál será necesario optar por un tipo de actor social que lleve a cabo la innovación requerida. Si lo lógico es que ese actor llamado a desencadenar nuevas metodologías y formas de aproximarse al conocimiento es el profesor, será necesario por tanto establecer cuál es el tipo de profesor que se requiere para la nueva educación y cuáles son las condiciones estructurales (organizacionales) y simbólicas (motivacionales) que se requieren para la transformación deseada.

\section{4. ¿CÓMO LAS TIC’S REPLANTEAN LA RELACIÓN ESCUELA-MUNDO?}

Uno de los puntos quizás centrales de la ideología que sustenta al proyecto Enlaces, refiere al supuesto de que la tecnología permitirá acercar el mundo (como espacio físico y simbólico) a los niños y jóvenes de los lugares más recónditos de Chile.

Tal idea cobra especial potencia, cuando nos situamos frente a una realidad tan particular como es la que viven las localidades de sectores rurales, casi siempre excluidas de los procesos de producción y 
desarrollo de tecnologías y nuevos conocimientos.

El hecho de que lo rural se constituya como un espacio tradicionalmente postergado de las innovaciones tecnológicas, se reafirma en las teorías clásicas de la antropología rural que sitúan a la ruralidad como un espacio opositivo y dependiente de lo urbano, sinónimo de modernidad y desarrollo. Si bien este planteamiento ha sido criticado en virtud de que se les resta importancia y autonomía a los procesos sociales que ocurren en lo rural, al considerarlo sólo como un residuo de lo tradicional y lo marginal frente a la centralidad de la urbe. No deja de cobrar cierto vigor esa idea de exclusión, si la discusión se pone en la perspectiva de lo que se ha llamado "brecha digital", es decir, ese quiebre entre las sociedades que han alcanzado un estándard alto en relación al uso de las nuevas tecnologías y el de sociedades que han ido quedando relegadas en ese proceso.

Ahora bien, las TIC's parecen plantear un nuevo escenario para la tensión entre lo local y lo global. Tensión que aparece latente en la escuela rural, por una parte institución enraizada en la cultura rural pero, a la vez, agente socializador de los marcos y normas del estado-nación. Esa posición de eje entre dos realidades (lo rural, y lo nacional) y su nuevo papel ante la llegada de las TIC's y sobre todo ante la potencialidades de Internet que sin duda relaciona a lo local y a lo global, constituye a la escuela en un espacio de negociación intercultural.

Este punto es especialmente significativo considerando que las escuelas rurales merced al proyecto Enlaces se transforman en el único referente informático comunicacional para comunidades muchas veces aisladas y con una reducida disponibilidad de telecomunicaciones. $Y$ pese a que el equipamiento tecnológico de las escuelas rara vez muestra niveles óptimos (en cuanto a conectividad, renovación de computadores, actualización de software, etc) sigue siendo el único lugar disponible en la comunidad. Este hecho marca un acercamiento y una aproximación gradual a las potencialidades de la tecnología. Dicho de un modo más directo, la escuela rural que forma parte de la "Red Enlaces", se constituye en la única forma de participar en los procesos comunicativos que dan forma a la globalización de la sociedad.

Un punto que reafirma lo anterior es que si bien lo que se produce en términos de aprendizaje y utilización de la tecnología es una familiarización tecnológica, traducida en una apropiación irregular y asimétrica de la tecnología, ya sea de profesores o alumnos, la escuela de igual forma logra situarse como un significativo, sino el único espacio tecnológico en el contexto de las comunidades rurales que han sido sistemáticamente excluidas ante el avance de los procesos de integración económica y social.

Al detenerse en lo anterior, se puede dimensionar que la escuela puede llegar a constituirse en el mediano plazo en un núcleo que permite la formación de un capital de conocimiento, que pese a ser discontinuo y precario, sigue siendo el único espacio que en estos momentos está entregando sistemáticamente, algún tipo de herramientas que permitan a los miembros de comunidades rurales lograr dimensionar los alcances e implicancias del proceso de transformación mundial que se manifiesta en la actualidad.

Los cuestionamientos que surgen son variados y complejos respecto a este tema, sin embargo se mueven, casi todos, en el plano de cuál debe ser la posición y el perfil de la escuela post-TIC's. Pues todo apunta a que la escuela para llevar a cabo el potencial transformador de la tecnología, lejos de adaptarla a sus propias dinámicas, parta por modificar su propia estructura y relaciones, y se resignifique como agente de socialización. Se trata de que se enfrente al punto de partida de un cambio de paradigma.

De este modo quedan abiertas como interrogantes ¿si la escuela podrá reposicionarse ante esta implosión y explosión comunicacional e informática que se desencadena en la localidad?, o ¿cómo las TIC's replantean a la escuela rural frente a la sociedad global?, ¿cuál es el papel que debe jugar la escuela como interlocutora y mediadora de la localidad ante los procesos complejos de la globalización? ¿cómo enfrentarse al reto de educar en un escenario cambiante que parece minar las certezas y los conocimientos con una rapidez jamás vista? ¿debe la escuela conformarse con educar a 
los alumnos o podrá capitalizar sus potencialidades de núcleo informático en la comunidad rural, abriéndole las puertas de la sociedad de la información a cada uno de sus actores?

\section{Propuestas}

\subsection{APROPIACIÓN CULTURAL DE LA TECNOLOGÍA.}

Durante esta investigación hemos sostenido que por sobre las consideraciones "de sentido común" que giran sobre las TIC's como herramientas introducidas en el sistema educacional y centrada en la dotación de equipos, existe un complejo campo de expectativas, roles, visiones de mundo, valores, etc que también juegan un papel fundamental en cuanto a la manera de comprender y utilizar las TIC's, que estos actores "beneficiados" evidencian. Todos estos elementos dan pie a un proceso de apropiación de la tecnología, el cual se expresa en las diversas valoraciones que hacen que (para el caso de nuestra investigación) profesores, directivos y alumnos de escuelas rurales tomen decisiones que los lleven a integrar progresivamente el uso de las TIC's a su quehacer más cotidiano o por el contrario desechar esta posibilidad y mantener las formas con las cuales tradicionalmente ha operado la escuela rural. Porque a nuestro juicio es en el plano de la apropiación cultural de la tecnología en donde se juega la viabilidad del proceso de introducción de las TIC, puesto que son los usuarios quienes deciden en definitiva qué tipo de uso y orientación entregarle a las nuevas tecnologías. Consideración que toma más fuerza aún si consideramos las rígidas estructuras que caracterizan a la cultura escolar.

Sin desconocer la evidente necesidad de contar con infraestructura informática que posibilite y entregue las facilidades mínimas para poder esperar la transformación en la educación que se promete a través de las TIC's; todo este esfuerzo adolece de una gran fragilidad en la medida en que no se considere la real importancia que los procesos culturales tienen a la hora de definir el éxito, el impacto y la profundidad de los cambios que se persiguen por medio de la generalización el uso de las TIC's.

Precisamente una de las formas de enfrentar esta debilidad para comprender y encauzar la política pública en cuestión se orienta a considerar a las variables (como expectativas, actitudes y usos) y sus respectivos indicadores dentro de una metodología que establezca la centralidad del concepto de apropiación, en tanto y cuanto, este posee un valor ambivalente que puede llegar a marcar el éxito o el fracaso de todo esfuerzo tendiente a mejorar la calidad de la educación a través de la integración del computador.

\subsection{METODOLOGÍA}

La constatación del hecho de que las TIC's son, hasta el momento, un elemento extraño a la escuela pone en juego la necesidad, por parte de esta, de elaborar una forma de trabajo y aprovechamiento de esta nueva tecnología. Las particulares características que las TIC's tienen como tecnología (la interacción, la velocidad, lo multimedial, etc.) exigen que se desarrollen estrategias pedagógicas diferentes a las que tradicionalmente han caracterizado a la escuela, como única forma de realmente poder aprovechar este desarrollo tecnológico para potenciar la educación.

Es por lo mismo que la posibilidad de integrar las TIC's a la dinámica escolar, lo que en definitiva es la condición para poder aprovecharlas en todo su potencial, no se logra solo con la presencia material de un computador si no que exige que se piense un método especifico de trabajarlas (por supuesto que en consonancia con el resto del curriculum), de ahí que sea pertinente hablar de una necesaria "metodología de integración pedagógica" de las TIC's al devenir pedagógico.

Esta metodología de trabajo con TIC's (que permita su integración provechosa) debe abarcar al menos dos ámbitos del mundo de la escuela. Por un lado el de la gestión escolar y, por otro, el del trabajo en el aula. En ambos casos las TIC's presentan un desafío, y a la vez se necesita de la concurrencia de ambos para generar la transformación pedagógica que se pretende con las TIC's. 
En el caso del ámbito de la gestión escolar la introducción de los computadores hace necesario desarrollar toda una estrategia nueva y especifica a los requerimientos que conlleva esta introducción de tecnología, que asuma las problemáticas y también las potencialidades que se generan en la dimensión institucional. Así por ejemplo las características técnicas de las TIC's exigen una permanente manutención y actualización, tanto de los software como de los hardware, esto por que por un lado es una tecnología relativamente delicada y por otro por que se va innovando constantemente, estableciendo nuevas fronteras de complejidad y capacidad de trabajo, por lo que requiere de una permanente inversión si es que se pretende dar real acceso al desarrollo tecnológico que esta viviendo el mundo (y no quedarse con el computador como una mera maquina de escribir) [10]. Estos requerimientos, por sus costos a nivel nacional, son mal asumidos por el organismo central (ministerio), que de hecho esta más preocupado por abarcar a nuevas escuelas que poder actualizar la tecnología ya existente, por lo mismo entonces la escuela, si quiere mantener la promesa de inserción tecnológica, debería empezar a generar recursos propios (ya sea dineros, asesorías, donaciones, etc.) para poder mantener un mínimo horizonte de utilidad de su infraestructura tecnológica.

A la vez la necesidad de buscar un mejor aprovechamiento institucional de las TIC's obliga a generar un ordenamiento de horarios que garantice efectivamente un acceso permanente de todos los alumnos y en todos los ramos, lo que pone en juego, por ejemplo la disponibilidad horaria de profesores. Lo anterior se relaciona con la necesidad de establecer una verdadera integración curricular (a nivel escuela) lo cual pasa, en parte importante, por crear las condiciones institucionales que la permitan como un objetivo real a conseguir.

Estos desafíos, y también sus potencialidades, que se presentan con la introducción de las TIC's en las escuelas obligan a pensar nuevos métodos y lógicas de trabajo, por que (si se quiere aprovechar al máximo sus potencialidades) la gestión en la escuela debe cambiar, ya que establecen nuevos desafíos que hace mucho tiempo no se generaban.

La integración de las TIC's en las escuelas también impacta en lo que al trabajo pedagógico en el aula se refiere, o sea en otras palabras, es vital repensar las formas de trabajar con las TIC's si se quiere conseguir un resultado pedagógico significativo.

En este punto la metodología de trabajo debe tomar en cuenta la realidad en que se sitúa la introducción de tecnología en estas escuelas, y esta es que los recursos no alcanzan para lograr un ideal de un computador por alumno, por lo que el primer dato de realidad para la construcción de una metodología de trabajo en el aula es la carencia en el número de computadores. De ahí que en primer término es necesario pensar una forma de trabajo grupal, la cual tenga como objetivo básico el garantizar que todo alumno asuma la interactividad con el computador[11]. Esto requiere a su vez que los software también asuman en su diseño una lógica de trabajo grupal.

Esta lógica grupal debe ser complementada con una dinámica mucho más participativa por parte de los alumnos, ya que la única forma en que se pueda aprovechar toda la potencialidad de las TIC's radica en que el alumno pueda ejercer plena interactividad con el computador. En otras palabras el alumnos tiene que poder ejercer una cierta "soberanía", tiene que tener espacio suficiente para desarrollar una interacción propia con el computador, por lo cual el profesor debe saber desenvolverse de una forma en que, manteniendo una orientación acorde con la asignatura, se constituya precisamente en un sujeto facilitador El profesor en este sentido debe generar en el alumno una preocupación, una motivación por utilizar el computador, por acceder a internet como una forma de potenciar su formación.

Este desafío por repensar el aula conlleva repensar a los actores que en el convergen, en este sentido, dadas las características mucho más dinámicas del trabajo con las TIC's, surge con particular importancia la necesidad de establecer estrategias que permitan en cierta medida integrar a los "acólitos"[12] en el trabajo con los computadores. Esto por que gran parte de la formación que se requiere para llegar a manejarse en las TIC's se sustenta en la motivación y cercanía que se pueda establecer con ellas, y son precisamente los "acólitos" quienes han podido desarrollar rápidamente 
tales características (además por supuesto de un dominio muchas veces superior al de la mayoría de los profesores), por lo cual se encuentran en una posición optima para potenciar la tarea de la escuela.

En definitiva es necesario tener en claro que hasta el momento existe un ámbito en cual la introducción de tecnología en las escuelas esta cojeando y que permite, en cierta medida, dar cuenta de las limitaciones del impacto pedagógico de este enorme esfuerzo publico. No basta la presencia de un computador para lograr potenciar los avances pedagógicos La clave esta en que el computador permite sustentar transformaciones que van más allá de el, ya que el verdadero sentido de estos avances pedagógicos se encuentra en la transformación metodológica, en la innovación respecto a las estrategias del aprendizaje.

\subsection{CAPACITACIÓN}

Todo desarrollo tecnológico necesita generar a la par un desarrollo humano (lo que en términos actuales se podría denominar como "recursos humanos"), en otras palabras el desarrollo de una tecnología no se encuentra separado de las transformaciones sociales que ella conlleva, por lo tanto toda innovación tecnológica requiere generar un recurso humano que la sustente. La invención material no vale nada por si misma, si no que solamente cuando adquiere un sentido y por ende genera transformaciones sociales.

Lo anterior se percibe claramente a la hora de observar el proceso de introducción de las TIC's en las escuelas, ya que por las características que estas tienen no son comparables a ninguna de las herramientas pedagógicas tradicionales[13], más aun si se toma en cuenta que la escuela, como institución, no ha enfrentado ninguna innovación tecnológica significativa en décadas. Esto da cuenta de que la escuela en realidad no contaba con los recursos humanos necesarios para poder implementar en toda su magnitud ese proceso tecnológico[14] y por ende aun hoy en día se enfrenta a la escasez de personal apto para llevar a cabo tamaña empresa. Es a esto a lo que apunta la capacitación que dan las universidades.

Ahora bien la gran falencia que en general presenta el mecanismo de capacitación que se ha venido implementando es que se centra solo en la dimensión técnica, en el saber usar (cómo prender, el uso del mouse, entrar a internet, etc.). Si bien es obvia la necesidad de enseñar a usar la tecnología cabe preguntarse si es que con esto es suficiente para formar los recursos humanos que se necesitan.

A la luz de lo observado es claro que no, y esto es por una razón muy sencilla, el saber la tecnología no la dota automáticamente de un sentido, el saber usar un computador no significa que dimensione todas sus potencialidades, ni tampoco genera, necesariamente, un afán por innovar. Es por esto que la capacitación, si es que pretende ser realmente una formadora de recursos humanos que sustenten la introducción de esta tecnología en la escuela, debe superar lo meramente técnico y abordar aspectos motivacionales e ideológicos. Esto quiere decir que la capacitación debe generar sentidos para el profesor (y también para el estrato administrativo), debe mostrarle las potencialidades que se esconden en el computador, debe entusiasmarlo con una nueva forma de trabajar, debe quitarle el miedo y el recelo que pueda sentir, en definitiva debe, en primer lugar, ser capaz de convencer al profesor, ya que solamente un profesor convencido y entusiasmado con este proyecto va a ser realmente útil, pedagógicamente hablando (como es el caso de los "encargados").

En este sentido la capacitación no debe estar solamente orientada al aula, si no que debe apuntar a abarcar integralmente al profesor como profesional e incluso como persona, en otras palabras si el no es capaz de ver la utilidad para si mismo es difícil que la asuma para los demás y por ende se reciente gravemente su capacidad para enseñarla. La capacitación entonces debe ser capaz de enseñar nuevas metodologías de trabajo, tanto para el aula como también para la gestión escolar, que le permitan al profesor percibir las oportunidades que se le pueden abrir. Lo anterior apunta en definitiva a la necesidad de generar un profesor de carácter "facilitador"'[15] que se constituya en el sujeto central de todo un proceso de innovación tecnológica, pero sobretodo metodológica dentro de las escuelas de este país. 
Para lograr lo anterior ciertamente es imprescindible acentuar la capacitación y esto obviamente implica invertir más en ella. Hay que invertir en serio, por que generar recursos humanos no es algo barato y pretender lograrlo con algún descuento es simplemente hipotecar todo el proceso, un lujo que a esta altura sería imperdonable.

\subsection{DEMOCRATIZAR LAS TIC A TRAVÉS DE LA APERTURA HACIA LA COMUNIDAD.}

Otro elemento que caracteriza a la implementación del proyecto Enlaces tiene que ver con una tendencia a entender la instalación de esta transformación tecnológica como un fenómeno pertinente sólo a la escuela. Si se toma en consideración los argumentos expuestos en este trabajo en torno a los conceptos de apropiación, brecha digital de $2^{\circ}$ orden, gestión, rápidamente se logra constatar que esta visión errada en donde el proceso de introducción de las TIC's es visto como un compartimiento estanco en la escuela solo dificulta la posterior integración de las TIC's. Ahora bien, parte de esta visión de corte más sistémico implica acercar estos nuevos recursos hacia la comunidad.

En buenas cuentas lo que ocurre con la escuela rural es que el diseño propuesto por Enlaces más los componentes propios de la cultura escolar hacen que se entienda a la introducción de las TIC's en ella como un proceso desligado de su entorno más inmediato que viene a ser la comunidad que en este caso es carácter rural. Mientras se piensa en la posibilidad de conectar a los alumnos con otros pares de lugares distantes se tiende a desaprovechar el rol que puede jugar la comunidad en esto, basta pensar en el juego de expectativas que hay entre los padres y sus hijos como elemento que puede motivar o desencantar a los alumnos en el uso de las TIC's. Si entendemos al proyecto Enlaces como una iniciativa de alfabetización y extensión tecnológica no hay razón para que esta no contemple alguna actividad que integre a la comunidad. Nuestra propuesta busca pesquisar que tan viable puede ser esta propuesta desde algunos componentes básicos que dan forma y sentido a la cultura escolar en áreas rurales, más aún cuando, a nuestro juicio, la presencia masiva de las TIC's no puede ser entendida automáticamente como sinónimo de apropiación cultural, mejoramiento de la calidad de la educación o de integración no conflictiva a la "sociedad de la información".

Como es sabido en la actualidad existe un número importante de escuelas rurales que ya cuentan con infraestructura de TIC's, algunas incluso durante un período de varios años. En este sentido es importante hacer notar que la escuela que cuenta con equipamiento en informática muchas veces se transforma en el único referente informático para comunidades muchas veces aisladas y con una reducida disponibilidad de telecomunicaciones. Pese a que el equipamiento tecnológico de estas escuelas rara vez muestra niveles óptimos (en cuanto a conectividad, renovación de computadores, actualización de software, etc) sigue siendo el único disponible en la comunidad lo que marca un acercamiento y una aproximación gradual a las potencialidades de la tecnología. Dicho de otro modo, la escuela rural que forma parte de la "Red Enlaces" se constituye en la única forma de participar en los procesos comunicativos que dan forma a la globalización de la sociedad

En buenas cuentas lo que se propone es ampliar aún más la noción de "objetivo transversal de la educación" como se define a los usos y aplicaciones de la informática. Rompiendo la función exclusivamente instructora (enseñanza a través del computador) que se le otorga a las TIC's y ampliando su acceso hacia la comunidad. De hecho, ya existen numerosas iniciativas en esta misma línea, como lo son la instalación de Infocentros de uso comunitario los cuales ya operan en diversos puntos del país. De la integración de juntas de vecinos y otras organizaciones de la sociedad civil a este "núcleo tecnológico" en que se constituye la escuela, lo que se espera no es sólo un acceso más igualitario y universal a estas tecnologías de información y comunicación sino que también la generación de sinergias que colaboren en el proceso, ya no de introducción sino de integración de las TIC's.

\section{Bibliografía}

Augé, M. Los "no lugares", espacios del anonimato: Una Antropología de la sobremodernidad. Gedisa, Barcelona, España. 1993. 
Berners-Lee, T. (1999). Weaving the Web: The Original Design and Ultimate Destinity of the World Wide Web by Its Inventor (1 st ed.): Harper San Francisco.

Bruckman, A. (2001). The Future of E-Learning Communities. Communications of the ACM, 45(4), 60-63.

Brunner, J. J. "Chile: Informe e índice sobre capacidad tecnológica.". Universidad Adolfo Ibáñez, Instituto de Economía Política. Santiago, 2001

Harel, I., \& Papert, S. (Eds.). (1991). Constructionism. Norwood, N J: Ablex Publishing.

Hiltz, S. R., \& Turoff, M. (2002). What makes learning Networks Effective? Communications of the ACM, 45(4), 56-59.

Hopenhayn, M. y Ottone, E. “El gran eslabón” ed. Fondo de Cultura Económica, Argentina, 2000.

Huizinga, Johan. Homo Ludens. Emecé editores, colección Piragua, Buenos Aires, 1968.

Johnson-Gentile, K., Lonberger, R., Parana, J., \& West, A. (2000). Preparing Preservice Teachers for the Technological Classroom: A School-College Partnership. Journal of Technology and Teacher Education, 8(2), 97-109.

Lull, James .Inside family viewing. Ed Routledge , Londres. Inglaterra. 1990.

Martín - Barbero, Jesús. De los medios a las mediaciones. Editorial Gustavo Gili, México, D.F 1987.

MINEDUC. (1998). Objetivos Fundamentales y Contenidos Mínimos Obligatorios de la Educación Media (1st ed.). Santiago: Ministerio de Educación Pública, Chile.

Morley, David. Televisión, audiencias y estudios culturales. Amorrortu Editores, Buenos Aires, Argentina. 1996.

Morley, David. Family television. Ed Routledge. Londres, Inglaterra. 1986.

Negroponte, Nicholas. Ser Digital. Ed Atlántida. Buenos Aires, Argentina. 1995.

Orozco, Guillermo. Televisión y audiencias. Un enfoque cualitativo. Universidad Iberoamericana, Ediciones de la Torre, Madrid, España. 1996.

Piscitelli, Alejandro. Ciberculturas En la era de las máquinas inteligentes. Ed Paidos, Buenos Aires, Argentina. 1996.

Rheingold, Howard. La comunidad virtual. Ed Gedisa. Barcelona, España. 1998.

Sunkel, Guillermo (ed). El consumo cultural en América Latina. Ed Andrés Bello. Santa Fe de Bogotá, Colombia. 1999.

Torres, R. M. (2001). La profesión docente en la era de la informática y la lucha contra la pobreza (Documento de Apoyo ED-01/PROMEDLAC VII/). Santiago: UNESCO.

Wenglinsky, H. (1998). Does It Compute? The Relationship Between Educational Technology and Student Achievement in Mathematics. Princeton: ETS Policy Information Center. 
[1] Enlaces. Red educacional. Ministerio de Educación de Chile, 2000. pág.: 11.

[2] Entrevista a José Joaquín Brunner en Informe de Desarrollo Humano Chile 2000. PNUD pág.: 214.

[3] Ubicada en la séptima región del Maule, está emplazada en la ribera sur del río de igual nombre a unos 280 kilómetros al sur de Santiago. La capital regional que es la ciudad de Talca se ubica a unos 20 kilómetros al norte, a sólo unos 15 minutos del principal centro poblado de la comuna. La comuna cuenta con un número de 13.769 habitantes, de los cuales 11.007 personas viven en asentamientos rurales mientras que sólo 2.762 se ubican en el único asentamiento 'urbano'.

[4] Lo más similar, y a lo cual se recurre muchas en la bibliografía especializada, ha sido los experimentos pedagógicos con la televisión y el video en el aula. Aun cuando tecnológicamente se puedan establecer ciertas similitudes entre la televisión y un computador, pedagógicamente hablando ambas experiencias son radicalmente distintas. Las TIC's asumen un muchísimo mayor potencial pedagógico y una promesa utópica de enormes magnitudes, que la televisión en la escuela nunca estableció.

[5] Aun cuando la definición de "nuevo" es bastante clara.

[6] Ciertamente este anhelo por la innovación no solamente una razón de ser discursiva si no que, por ejemplo, relaciona mucho con la forma en que se concibe el desenvolvimiento de la economía.

[7] De ahí la necesidad de establecer una tipología de carácter "ideal" para poder dar cuenta del comportamiento de los profesores ante las TIC's. Ver capitulo 1.

[8] Extraño más que en una acepción de no conocido, en un sentido de ajeno, ya que puede ser que pasado el tiempo los computadores sean conocidos por los profesores (que incluso sepan manejarlos), pero si estos (y sus alumnos) no los sienten como algo propio e imprescindible para el proceso educativo van a seguir siendo ajenos (traídos desde fuera; como un lujo prescindible; como algo reservado para algunos pocos interesados, etc.)

[9] Abarcando esencialmente a los "acólitos" y los encargados y centrada en la Sala de Computación.

[10] Esto se percibe claramente en la Internet, la cual exige cada vez mayores capacidades de los computadores para poder acceder a sus potencialidades, como por ejemplo desde bajar música hasta crear páginas web.

[11] A diferencia con lo que sucede hoy día en que solo uno o dos del grupo se apropian del computador relegando a los otros a solo mirar.

[12] Véase capítulo respecto a la Sala de Computación como lugar sagrado.

[13] El comparar a las TIC's con el pizarrón implica desnaturalizarlas.

[14] Los cuales son incluso escasos a nivel de la sociedad en general.

[15] Ver respecto a las tipologías de profesores.

(C) Ediciones Universidad de Salamanca 